\title{
Lamborghini Aventador SVJ Aerodynamics: Route to breaking the super sport car's record
}

\author{
Ugo Riccio, A. Torluccio
}

Automobili Lamborghini S.p.A.

This manuscript is not available according to publishing restriction.

Thank you for your understanding. 